\title{
Article
}

\section{An evaluation of Fracture Liaison Services in the detection and management of osteoporotic fragility fractures: a narrative review}

Eccles, E, Thompson, J and Roddam, Hazel

Available at http://clok.uclan.ac.uk/22772/

Eccles, E, Thompson, J and Roddam, Hazel ORCID: 0000-0002-0637-1801

(2018) An evaluation of Fracture Liaison Services in the detection and management of osteoporotic fragility fractures: a narrative review.

Radiography, 24 (4). pp. 392-395. ISSN 1078-8174

It is advisable to refer to the publisher's version if you intend to cite from the work. http://dx.doi.org/10.1016/j.radi.2018.05.003

For more information about UCLan's research in this area go to http://www.uclan.ac.uk/researchgroups/ and search for <name of research Group>.

For information about Research generally at UCLan please go to http://www.uclan.ac.uk/research/

All outputs in CLoK are protected by Intellectual Property Rights law, including Copyright law. Copyright, IPR and Moral Rights for the works on this site are retained by the individual authors and/or other copyright owners. Terms and conditions for use of this material are defined in the policies page. 


\section{An evaluation of Fracture Liaison Services in the detection and management of osteoporotic fragility fractures: a narrative review}

\section{Introduction}

Osteoporosis is a bone disease characterised by a loss of bone density and deterioration in bone structure. For patients with osteoporosis, the resilience of the bone is compromised, placing the patient at an increased risk of a fragility fracture. Osteoporosis is often referred to as a 'silent disease', where the patient is often asymptomatic until a fracture occurs. Fragility fractures are defined as those which occur from low-impact mechanical forces that would not normally result in fracture; these are due to low bone density and structural deterioration of bone tissue. The World Health Organisation (WHO) has quantified these forces as being equivalent to those experienced from a fall from standing height or less. ${ }^{1}$

This type of injury can have a very poor prognosis, with fragility fractures of the hip and spine associated with increased 5 -year mortality rate. A recent study has revealed that $24 \%$ of women and $20 \%$ of men re-fractured, and $26 \%$ of women and $37 \%$ of men died without refracture in this 5 -year period. Of those who re-fractured, a further $50 \%$ of women and $75 \%$ of men died, resulting in a total 5 -year mortality rate of $39 \%$ in women and $51 \%$ in men. ${ }^{2}$ An added complication is that approximately $50 \%$ of people with a fragility fracture will suffer another. Warning signs do exist for this patient group, as almost half of those that present with a hip fracture will have suffered a previous fragility fracture, ${ }^{3}$ thus highlighting the missed opportunities to identify and treat this population.

This paper presents a synthesis of the current evidence base for the detection and management of osteoporotic fragility fractures, including Fracture Liaison Service initiatives. A systematic approach was undertaken to identify relevant sources, charting the key findings to generate an integrative narrative review and highlighting implications for future commissioning and service delivery. 


\section{An ageing population, a cost to society}

Large increases in life expectancy are being observed in the majority of developed countries. As a consequence, the number of fractures in the elderly population is expected to increase. ${ }^{4}$ In Australia it is expected that $66 \%$ of those aged over 50 will be affected by osteoporosis. ${ }^{5}$ This is similar to the UK, where osteoporosis and the associated consequences are becoming ever more prevalent, with 1 in 2 women and 1 in 5 men expected to have a fragility fracture after the age of $50 .{ }^{1}$ The synergy of population ageing and osteoporosis will become an economic burden. ${ }^{6,7}$ The human cost is more difficult to measure, but the co-morbidities and potential mortality rate associated with an ageing population are not difficult to appreciate. With this in mind, it is crucial that secondary prevention becomes a core characteristic in the management of fractures and osteoporosis. ${ }^{8}$

Cost is always at the forefront of discussion of service delivery and provision in the UK National Health Service (NHS), and this is no different in the care of osteoporosis and fragility fractures. Osteoporotic fragility fractures are costly both in human and economic terms. Over 300,000 patients present with fragility fractures to hospitals in the UK each year. Social and medical costs from fragility fractures to the UK healthcare economy were estimated at $£ 1.8$ billion in 2000 , with much of this due to fractures of the hip. ${ }^{8}$ Due to an aging population this has the potential to increase to $f 2.2$ billion by 2025 , with most of these costs relating to the ongoing care of fragility fractures to the hip, spine, and wrist. ${ }^{9}$ Hip fractures are the most significant type of fragility fracture because of the human impact and the need for long-term institutional care, and associated high medical costs. ${ }^{10}$ The expected level of cost is not unique to the UK, where the cost of osteoporosis and fracture care in Australia is expected to rise to 3.84 billion Australian Dollars ( $£ 2.2$ billion) by $2020 .^{5}$ Nakayama et al $^{7}$ paint a similarly bleak worldwide picture in terms of a projected cost of 37 billion Euros in Europe by 2025 and 12.5 billion US Dollars in China by 2020. Despite these large costs, the rate of investigation and treatment of osteoporosis remains low. However, a recent technology appraisal of bisphosphonate treatment for osteoporosis by the National Institute for Health and Care Excellence (NICE) ${ }^{11}$ aimed to establish at what level of absolute fracture risk the treatments are cost effective. A reduction in price means that bisphosphonates are now cost effective even in patients with a low level absolute risk (i.e. oral medication should be considered for those with $>1 \%$ risk of fracture in a 10 -year 
period). The treatment regime should be based on individuals (rather than populations) in order to understand relative advantages and disadvantages. For those patients considered higher risk ( $>10 \%$ risk of fracture in a 10 -year period), or those who cannot tolerate oral medications, it is appropriate to offer intravenous bisphosphonates. This new cost assessment should encourage the use of bisphosphonate treatment in patients who are at risk of fragility fracture.

\section{Fragility fracture identification: the role of radiology}

Positive identification of fragility fractures is critical to the care of patients with osteoporosis. Vertebral fractures are the most common type of fragility fracture, accounting for almost half of all fractures due to osteoporosis. ${ }^{12,13}$ They are a significant health concern due to the increased risk of future fractures and an associated increase in morbidity and mortality. ${ }^{14,15}$ In this patient group, a previous fracture is thought to double the risk for subsequent fractures, and in the case of vertebral fractures this risk is quadrupled. ${ }^{4}$ The risk of vertebral fracture increases exponentially with a greater number of prior vertebral fractures; known as the vertebral fracture cascade. As a further complication the risk of hip fracture is also doubled. ${ }^{13,16}$

Despite the clinical significance of vertebral fractures, these are often overlooked clinically and/or radiologically, with many studies demonstrating that the under-reporting of vertebral fractures is a world-wide problem. ${ }^{14,16-18}$ In Europe, around $1 / 3^{\text {rd }}$ of vertebral fractures are overlooked and this has been attributed to asymptomatic presentation, lack of radiographic detection and ambiguous terminology in the radiological report (i.e. wedging, vertebral height loss, deformity, or end-plate infraction or depression). ${ }^{13,14}$ Even when they are detected in hospital, it does not necessarily mean that it will lead on to an assessment of bone health or subsequent treatment. ${ }^{15}$

In the clinical setting the asymptomatic nature of vertebral fractures presents a challenge, meaning that they can go untreated and the future fracture risk remains. So how can radiology help? 
The National Osteoporosis Society (NOS) has provided recent guidance ${ }^{19}$ about the detection of vertebral fractures, where it explains the critical role of diagnostic imaging services. Radiology practitioners are often best placed to initiate the most substantial improvements in the vertebral fracture pathway - starting with effective detection of fractures. The NOS has recommended that local protocols are established to ensure that the spine is routinely evaluated for the presence of vertebral fractures of the thoracic and lumbar spine on any imaging, regardless of the clinical question. Upon identification of a fracture the referring clinician should be alerted, using a fail-safe mechanism, so that they understand need to investigate the patient for fracture risk. It is crucial that unambiguous terminology is used and the NOS has recommended that the condition of the vertebral body should be clearly described in one of three ways: (i) vertebral fracture (including level, severity and timing), (ii) non-fracture vertebral deformity (i.e. Scheuermann's disease or Schmorl's nodes), and (iii) normal. ${ }^{19}$ The NOS also provide suggested standard phrases that could be used in a radiological report.

In addition to the targeted identification proposed by the National Osteoporosis Society, reporting practitioners should always be aware of the opportunistic chance to identify fragility fractures. Incidental diagnosis of fragility fractures can often be made on computed tomography (CT) scans of the thorax and abdomen. Multi-Detector CT (MCDT) midline sagittal images can routinely be reformatted (without additional radiation dose to the patient) to help identify fragility fractures. Sagittal reformats are particularly sensitive for identification of vertebral fractures due to good visualisation of the middle of the end plate, where insufficiency fractures typically occur. ${ }^{16,20}$

Although the clinical significance of vertebral fractures is understood and the importance of opportunistically identifying such fractures has been recognised, there is still significant under-reporting. Widespread underreporting occurs in all imaging techniques, with MDCT missing more opportunities than radiographic imaging. ${ }^{16}$ Standardisation of the radiological assessment of vertebral fractures is also required, where clinicians often fail to recognise or report mild to moderate vertebral fractures, or use terminology that is not specific for fracture..$^{20}$ Effective communication to the wider team caring for these patients is critical to ensure that eligible patients receive either dual-energy X-ray absorptiometry (DXA) or pharmacologic therapy to reduce future fracture risk. ${ }^{20,21}$ Correct identification of fragility 
fractures that leads onto suitable investigation is the key improvement required for secondary prevention. ${ }^{15}$

\section{Fracture Liaison Services: a long-term solution, not a quick fix}

To reduce the risk of re-fracture a co-ordinated approach is needed to identify patients most at risk. A Fracture Liaison Service (FLS) is a proven approach to delivering comprehensive secondary prevention, which requires a multidisciplinary approach to be an effective service. This should comprise osteoporosis assessment and treatment together with a falls risk assessment. Ideally this would occur in a streamlined 'one-stop shop' setting. However, the challenge of organising such services and integrating them across acute and primary care is considerable. ${ }^{22} \mathrm{~A}$ raised awareness of such services must be a priority.

Cost appears to be a significant factor affecting the development of FLS within the NHS, but it is the longer-term cost-saving and patient benefit that needs to be considered. The financial value of FLS is based on reducing fragility fractures in a population. In 2009 the Department of Health provided an economic evaluation of fracture prevention services, where it was suggested that $£ 8.5$ million could be saved nationally over a 5 year period..$^{23}$ Despite this, such services are not universally adopted and this may in part be due to more modest estimations of cost saving. ${ }^{24}$ There is some evidence that a FLS can be effective when comparing small cohorts of patients that are treated against those that are not, but demonstrating financial value in an entire population is a challenge. It is suggested that demonstrating value of a FLS at a statistically significant level is impossible in a 1-3 year period, over which the financial value of a service is typically evaluated. ${ }^{25}$ Furthermore it has also been implied that current commissioning and budgeting processes within the NHS do not incentivise this type of service: when Sutcliff ${ }^{8}$ made this suggestion in 2008 a FLS was available in only $30 \%$ of NHS institutes. Nevertheless, investing in FLS should ultimately lead to lower overall healthcare costs, though the cost-effectiveness of each FLS is dependent on the structure, context, and geographical nature of the service. ${ }^{15}$ It is therefore important to establish what constitutes an effective FLS model of care. UK national clinical standards for FLS have been published by the National Osteoporosis Society (NOS) $51 \mathrm{Q}$ model. ${ }^{25}$ 
Other models of care have been suggested, and will be considered later in this paper, but a FLS is recognised as the current model of best practice. ${ }^{8}$ The main purpose of a FLS is to correctly identify patients with fragility fractures and subsequently provide them with appropriate follow-on treatment and assessment of bone health and future fracture risk. ${ }^{7,15}$ For all patients the aim is to extend care beyond healing of the first fracture and aim to reduce the risk of subsequent fractures, in particular those that are associated with higher morbidity (i.e. hip, vertebral)..$^{8,15}$

The British Orthopaedic Association set out six standards for hip fracture care. Standard 5 states that all patients presenting with fragility fracture should be assessed to determine their need for anti-resorptive therapy to prevent future osteoporotic fractures. ${ }^{22}$ A FLS should be applied to all patients of $\geq 50$ years and older who present with fragility fractures ${ }^{8,22}$ and it should also target patients with a previous history of fracture. This targeted treatment via implementation of a FLS is associated with better diagnosis of osteoporosis and a reduction in the number of secondary fractures. ${ }^{5}$

A FLS based within an acute setting serves to improve care for patients who present with a low impact (fragility) fracture i.e. gained from a fall, slip or trip. Patients should receive a clinical assessment by a specialist FLS coordinator or specialist nurse, and some patients will also undergo DXA bone density measurements at the spine and hip (in accordance with NICE guidance TA161). Treatment for osteoporosis is typically recommended in about $75 \%$ of these cases. $^{23}$ There is an now an acceptance that FLS should be the standard of care, and not an optional extra. ${ }^{25}$

\section{A gap in care}

The adoption of FLS is not universal worldwide, or even throughout the NHS. Reduced or alternative models have been implemented, with reliance on referral letters being sent to primary care physicians in general practice or to endocrinologists. Those systems have been found to be less effective than FLS, which leads the way in terms of diagnosis and treatment to prevent secondary fracture. ${ }^{26}$ These less intensive models that focus on improving knowledge of bone health have not yielded positive results in terms of re-fracture rate, ${ }^{15}$ 
while services that are reliant on letters to primary care result in low prescription rates of bisphosphonates. ${ }^{25}$ In addition, community based models suffer through low attendance rates ( $\sim 5 \%$ ) for follow-on DXA. ${ }^{25}$ In addition, DXA alone is not sufficient to provide an accurate prediction of fracture risk. A range of other non-bone mineral density related risk factors are required to accurately estimate the probability of fracture. ${ }^{4,15}$ Studies from the UK suggest that only one third of patients with a fracture undergo osteoporosis risk assessment and subsequent treatment for secondary prevention of fracture, ${ }^{22,27}$ despite the fact that it is recommended that a FLS should be available in all hospitals that provide definitive fracture care.

A study by Nakayama et $\mathrm{al}^{7}$ compared the outcomes for patients in hospitals with and without a FLS. Those patients treated at a hospital with a FLS were less likely to experience major and minor secondary fractures. Walters et $\mathrm{al}^{15}$ also report on a significant reduction in secondary fractures over a 2-4 year period when using a FLS compared to primary care follow-up or hospitals that did not use FLS. Cases of poor post fracture care have been attributed to a lack of leadership by any single profession, ${ }^{26}$ thus highlighting the need for a robust service with competent communication channels. When separate professional groups are left to treat the patients independently, instead of following a proper model, the overall care can suffer despite the best efforts of those involved. Mitchell et $a^{28}$ report that while orthopaedic surgeons are capable of delivering expert care for acute injury, they may not be the best at initiating the appropriate follow-up to investigate and treat the primary cause (i.e. osteoporosis). The same is true in primary care where the general practitioners may not instigate treatment unless it is recommended in a discharge summary by a specialist practitioner. Furthermore, a hospital based specialist may also not deliver the best care if their actions are not captured by a robust system supported by other professional groups, as would be found in a FLS. There are also reports of misrepresentation of DXA results, where multiple findings from the same examination are not successfully recorded due to a lack of compatibility of computer programs in the primary care setting. ${ }^{29}$

This is not to say that all FLS are infallible, as was found in an Australian study. Although compliance with national standards related to osteoporosis were improved alongside patient satisfaction, only $61 \%$ of patients went on to receive therapy that was specific to osteoporosis. This was thought to be because the presentation of fracture was not always 
typical, the degree of trauma appeared consistent with injury, or the patient refused treatment. On a positive note, based on their data it was predicted that secondary fractures would reduce by $16 \% .^{5}$

Orthogeriatric services (OGS) often run alongside a FLS in order to help with the care of elderly patients who are admitted to hospital following fracture. These patients are often too frail to attend outpatient appointments and OGS is valuable for the secondary prevention of fractures in these cases. ${ }^{30}$ High-quality and standardized care requires the development of specific local protocols based on national guidelines, to ensure clear directives on the delivery of post fragility fracture care. There is an ongoing debate among service leaders about the best way to organize and deliver a FLS. Currently, many primary care providers and acute trusts lack a systematic, case-finding approach to identify and treat osteoporosis in high-risk groups (i.e. post-menopausal women who have sustained a fragility fracture).

The introduction of secondary prevention of fragility fractures within the NHS Quality and Outcomes Framework (QOF) intends to incentivise the implementation of services in therapeutic domains, such as the care of fragility fractures. ${ }^{28}$ The QOF initiative is aimed at improving the standards in primary care by focusing on the results achieved in general practice. It rewards management of chronic conditions, major public health concerns and the implementation of preventative measures; so it is not difficult to appreciate how the care of fragility fractures and those with osteoporosis can fit this model. The NICE QOF indicators for this area are NM29, NM30 and NM31. ${ }^{31}$ These are based on accurate record keeping of patients who have a confirmed diagnosed of osteoporosis and have sustained a fragility fracture (NM29), and those with fragility fractures who are currently being treated with bone sparing agents (NM30/NM31). The categories are dependent on age, the timing of the fracture, and whether the diagnosis was made using DXA. ${ }^{31}$ The 'point' scoring model employed by QOF suggests a small improvement for the management of patients with osteoporosis from $2015 / 16$ to $2016 / 17$ but it may be too early to state this as a success. QOF has prompted improvements in care in the early years following implementation, but there is a persistant worry that once the pay for performance incentive expires, the quality of care may decline. ${ }^{32}$ In addition, there is no clear evidence to suggest that this improves patient outcomes, ${ }^{33}$ or that it is cost-effective compared to other methods of improving care. ${ }^{34}$ 


\section{Conclusion}

The financial value of FLS is based on reducing fragility fractures and the investment should reduce the cost of ongoing care in a population. However, the initial cost of implementation appears to be a significant factor affecting the adoption of FLS within the NHS despite the service being associated with better diagnosis of osteoporosis and a reduction in the number of secondary fractures.

Radiology has a key role to play in the positive identification of fragility fractures to ensure optimal, patient-centric care. To improve case finding of vertebral fractures, diagnostic imaging departments must follow fail-safe mechanisms and reporting guidelines to ensure all patients with fragility fracture are captured and cared for. The reduced cost of bisphosphonate treatment may potentially help to achieve longer term reductions in fragility fractures.

\section{References}

1. IOF bone health: What is osteoporosis. https://www.iofbonehealth.org/what-isosteoporosis. Published 2017.

2. Bliuc D, Nguyen ND, Nguyen T V, Eisman J a, Center JR. Compound risk of high mortality following osteoporotic fracture and refracture in elderly women and men. $J$ Bone Miner Res. 2013;28(11):2317-2324. doi:10.1002/jbmr.1968

3. Åkesson K, Marsh D, Mitchell PJ, et al. Capture the Fracture: A Best Practice Framework and global campaign to break the fragility fracture cycle. Osteoporos Int. 2013;24(8):2135-2152. doi:10.1007/s00198-013-2348-z

4. Huntjens KMB, Van Geel TACM, Blonk MC, et al. Implementation of osteoporosis guidelines: A survey of five large fracture liaison services in the Netherlands.

Osteoporos Int. 2011;22(7):2129-2135. doi:10.1007/s00198-010-1442-8 
5. Yates CJ, Chauchard MA, Liew D, Bucknill A, Wark JD. Bridging the osteoporosis treatment gap: Performance and cost-effectiveness of a fracture liaison service. J Clin Densitom. 2015;18(2):150-156. doi:10.1016/j.jocd.2015.01.003

6. Mueller D, Econ H, Gandjour A. Cost-effectiveness of using clinical risk factors with and without DXA for osteoporosis screening in postmenopausal women. Value Heal. 2009;12(8):1106-1117. doi:10.1111/j.1524-4733.2009.00577.x

7. Nakayama A, Major G, Holliday E, Attia J, Bogduk N. Evidence of effectiveness of a fracture liaison service to reduce the re-fracture rate. Osteoporos Int. 2016;27(3):873879. doi:10.1007/s00198-015-3443-0

8. Sutcliffe A. Osteoporosis: an overview of the organisation of services to deliver secondary prevention measures to older people with fragility fractures. Curr Orthop. 2008;22(5):315-321. doi:10.1016/j.cuor.2008.10.008

9. US Department of Health and Human Services. Bone health and osteoporosis: a report of the Surgeon General. US Heal Hum Serv. 2004:437. doi:10.2165/00002018200932030-00004

10. Papadimitriou N, Tsilidis KK, Orfanos $\mathrm{P}$, et al. Burden of hip fracture using disabilityadjusted life-years: a pooled analysis of prospective cohorts in the CHANCES consortium. Lancet Public Heal. 2017;2(5):e239-e246. doi:10.1016/S24682667(17)30046-4

11. National Institute for Health and Care Excellence: Bisphosphonates for treating osteoporosis [Technology Appraisal, TA464].

12. Chen P, Krege JH, Adachi JD, et al. Vertebral fracture status and the World Health Organization risk factors for predicting osteoporotic fracture risk. J Bone Miner Res. 2009;24(1523-4681 (Electronic)):495-502. doi:10.1359/jbmr.081103

13. Briggs AM, Greig AM, Wark JD. The vertebral fracture cascade in osteoporosis: A review of aetiopathogenesis. Osteoporos Int. 2007;18(5):575-584. doi:10.1007/s00198-006-0304-x

14. Pongchaiyakul C, Nguyen ND, Jones G, Center JR, Eisman JA, Nguyen T V. 
Asymptomatic vertebral deformity as a major risk factor for subsequent fractures and mortality: a long-term prospective study. J Bone Miner Res. 2005;20(8):1349-1355. doi:10.1359/JBMR.050317

15. Walters S, Khan T, Ong T, Sahota O. Fracture liaison services: Improving outcomes for patients with osteoporosis. Clin Interv Aging. 2017;12:117-127. doi:10.2147/CIA.S85551

16. Adams JE. Opportunistic Identification of Vertebral Fractures. J Clin Densitom. 2016;19(1):54-62. doi:10.1016/j.jocd.2015.08.010

17. Gehlbach SH, Bigelow C, Heimisdottir M, May S, Walker M, Kirkwood JR. Recognition of vertebral fracture in a clinical setting. Osteoporos Int. 2000;11:577-582. doi:10.1007/s001980070078

18. Williams AL, Al-Busaidi A, Sparrow PJ, Adams JE, Whitehouse RW. Under-reporting of osteoporotic vertebral fractures on computed tomography. Eur J Radiol. 2009;69(1):179-183. doi:10.1016/j.ejrad.2007.08.028

19. Adams J, Clark EM, Clunie G, et al. Clinical Guidance for the Effective Identification of Vertebral Fractures.; 2017. https://nos.org.uk/media/99100/vertebral-fracturesguidelines.pdf.

20. Adams JE, Lenchik L, Roux C, Harry K. Genant. Vertebral Fracture Initiative Part II Radiological Assessment of Vertebral Fracture.; 2006.

https://www.iofbonehealth.org/sites/default/files/PDFs/Vertebral Fracture Initiative/IOF_VFI-Part_II-Manuscript.pdf.

21. National Institute for Health and Clinical Excellence. Alendronate, etidronate, risedronate, raloxifene and strontium ranelate for the primary prevention of osteoporotic fragility fractures in postmenopausal women ( amended ). Natl Inst Heal Clin Excell. 2011;2008(January):94. doi:10.1016/j.semnephrol.2007.01.002

22. Marsh D, Colin C, Brown P, et al. The Care of Patients with Fragility Fracture. Blue B. 2007;(September):1-80. doi:10.1136/bmj.2.5211.1518

23. Department of Health. Fracture Prevention Services: An Economic Evaluation.; 2009. 
24. McLellan AR, Wolowacz SE, Zimovetz EA, et al. Fracture liaison services for the evaluation and management of patients with osteoporotic fracture: A costeffectiveness evaluation based on data collected over 8 years of service provision. Osteoporos Int. 2011;22(7):2083-2098. doi:10.1007/s00198-011-1534-0

25. Shipman KE, Doyle A, Arden H, Jones T, Gittoes NJ. Development of fracture liaison services: What have we learned? Injury. 2017:6-11. doi:10.1016/j.injury.2017.08.030

26. Noordin S, Allana S, Masri BA. Establishing a hospital based fracture liaison service to prevent secondary insufficiency fractures. Int J Surg. September 2017:1-5. doi:10.1016/j.ijsu.2017.09.010

27. Elvey MH, Pugh H, Schaller G, Dhotar G, Patel B, Oddy MJ. Failure in the application of fragility fracture prevention guidelines. Ann R Coll Surg Engl. 2014;96(5):381-385. doi:10.1308/003588414X13946184901164

28. Mitchell PJ, Chem C. Secondary prevention and estimation of fracture risk. Best Pract Res Clin Rheumatol. 2013;27(6):789-803. doi:10.1016/j.berh.2013.11.004

29. Chan T, De Lusignan S, Cooper A, Elliott M, Dore R. Improving osteoporosis management in primary care: An audit of the impact of a community based fracture liaison nurse. PLoS One. 2015;10(8):1-14. doi:10.1371/journal.pone.0132146

30. Mitchell P, Åkesson K, Chandran M, Cooper C, Ganda K, Schneider M. Implementation of Models of Care for secondary osteoporotic fracture prevention and orthogeriatric Models of Care for osteoporotic hip fracture. Best Pract Res Clin Rheumatol. 2016;30(3):536-558. doi:10.1016/j.berh.2016.09.008

31. NICE. NICE Quality and Outcomes Framework indicator. Quality and Outcomes Framework indicator. https://www.nice.org.uk/standards-andindicators/qofindicators?categories=3905\&page=1. Published 2017.

32. Roland M, Campbell S. Successes and Failures of Pay for Performance in the United Kingdom. N Engl J Med. 2014;370(20):1944-1949. doi:10.1056/NEJMhpr1316051

33. Mendelson A, Kondo K, Damberg C, et al. The effects of pay-for-performance programs on health, health care use, and processes of care: A systematic review. Ann 
Intern Med. 2017;166(5):341-353. doi:10.7326/M16-1881

34. Scott A, Sivey $P$, Ait Ouakrim D, et al. The effect of financial incentives on the quality of health care provided by primary care physicians. Cochrane Database Syst Rev. 2011;9(9):CD008451. doi:10.1002/14651858.CD008451.pub2 